\title{
Phenotypic and molecular identification of Sporothrix isolates of clinical origin in Northeast China
}

\author{
Xiaohong Yu • Zhe Wan • Zhenying Zhang • \\ Fuqiu Li $\cdot$ Ruoyu Li $\cdot$ Xiaoming Liu
}

Received: 30 November 2012/ Accepted: 31 May 2013/Published online: 16 June 2013

(C) The Author(s) 2013. This article is published with open access at Springerlink.com

\begin{abstract}
Sporotrichosis is the most common deep mycosis in Northeast China which is an area of high epidemicity due to contact with reeds or cornstalks. In this study, we have characterized a total of 74 clinical isolates from fixed cutaneous, lymphocutaneous and disseminated clinical forms and from Heilongjiang, Jilin, and Liaoning provinces, respectively. All isolates (previously as Sporothrix schenckii) were identified as Sporothrix globosa according to their phenotypic characteristics and calmodulin gene sequences analysis. They were subdivided into two sub-clades (S. globosa I and S. globosa II). Most of our isolates $(71 / 74)$ presented restricted growth at $37{ }^{\circ} \mathrm{C}$, which differed from a previous report. Up to now,
\end{abstract}

X. Yu $\cdot$ Z. Zhang $\cdot$ X. Liu $(\bowtie)$

Department of Dermatology and Venerology, The 1st Affiliated Hospital of Dalian Medical University, No.222, Zhongshan Road, Dalian 116011, China

e-mail: 1xm_1956@yahoo.com.cn

Z. Wan $\cdot$ R. Li $(\bowtie)$

Department of Dermatology and Venerology, Centre for Medical Mycology and Mycoses, The First Hospital, Peking University, No.8, Xishiku Street, Xicheng District, Beijing 100034, China

e-mail: 1ry0660@gmail.com

F. Li $(\bowtie)$

Department of Dermatology and Venerology, The Second Hospital of Jilin University, No.218, Ziqiang Street,

Changchun 130041, China

e-mail: lifuqiu@medmail.com.cn
S. globosa is the only pathogenic species in Northeast China, no matter what kind of clinical form and which region it is isolated from. Most of our clinical isolates (68/74) were clustered with three Chinese environmental isolates reported in the literature. The new findings of $S$. globosa isolates on division and thermotolerance at $37{ }^{\circ} \mathrm{C}$ described in this study will help us gain a better understanding of $S$. globosa.

Keywords Sporotrichosis - Sporothrix globosa . Calmodulin gene $\cdot$ Northeast China

\section{Introduction}

Sporotrichosis is a common subcutaneous mycosis which is caused by the dimorphic fungus previously described as the single species Sporothrix schenckii, now being recognized as Sporothrix complex which comprises at least six sibling phylogenetic species: S. pallida, S. brasiliensis, S. globosa, S. luriei, S. mexicana, and S. schenckii [1, 2]. Furthermore, S. pallida, S. nivea, and S. albicans were proposed to be synonyms since all the three species showed a considerably high genetic similarity [3]. Among the species of Sporothrix complex, there are differences in the geographical distributions. S. brasiliensis is restricted geographically to Brazil, and S. mexicana to Mexico [1]. Conversely, S. globosa is a widespread species found up to now in UK, Spain, Italy, China, Japan, USA, India, Mexico, Guatemala, Colombia, and more recently Brazil $[1,4,5]$. 
Sporothrix globosa species in China, which have been reported in the literature, are isolates from wheat, reed, and soil [6]. Which Sporothrix species (of clinical origin) being prevalent in China is still unknown to us. Northeast China including Heilongjiang, Jilin, and Liaoning provinces is the most endemic region [7-9] where sporadic cases have occurred for many decades, particularly in rural areas, with a high incidence in autumn and winter when the chances of contact with cornstalks increase. In recent years, small outbreaks have occurred in Jilin province with a significant increase in sporotrichosis.

The aim of this study was to investigate phylogenetic species of the Sporothrix complex in these areas and their morphological and physiological features, and to determine whether different clinical forms are associated with different species and whether outbreaks in Jilin province are caused by more virulent species than sporadic cases in Heilongjiang and Liaoning provinces.

\section{Materials and methods}

Fungal isolates

Seventy-four clinical isolates from human sporotrichosis patients were included in this study. All isolates were identified as $S$. schenckii by traditional morphological identification methods [3]. Forty-two of the isolates $\left(\mathrm{SHJU}_{1-40} / \mathrm{FHJU}_{1} / \mathrm{FHJU}_{2}\right)$ were from Jilin province, ten of the isolates $\left(\mathrm{HMU}_{1-10}\right)$ were from Heilongjiang province, and the remainder $\left(\mathrm{DMU}_{1-22}\right)$ were from Liaoning province. The cases from Heilongjiang and Liaoning provinces were sporadic between 1998 and 2012, and the cases from Jilin province were outbreaks during 2010 and 2011. Of all isolates, three isolates (DMU1/FHJU1/FHJU2) were disseminated clinical form, 39 isolates $\left(\mathrm{DMU}_{2-12} /\right.$ $\mathrm{HMU}_{1-5} / \mathrm{SHJU}_{18-40}$ ) were lymphocutaneous clinical form, and 32 isolates $\left(\mathrm{DMU}_{13-22} / \mathrm{HMU}_{6-10} / \mathrm{SHJU}_{1-17}\right)$ were fixed cutaneous clinical form. AM398393/AM39 8392/AM398396/AM747302/AM116899/AM117437/ AM399018/AM490354/AM399004/AM399002/GU4 56632/AM116908/AM398994/AM490358/AM3990 05/AM399015 were also included in phylogenesis analysis (Table 1).
Morphological studies

In order to study macroscopic features and thermotolerance (10), all isolates were subcultured on Potato Dextrose Agar (PDA-Difco ${ }^{\mathrm{TM}}$ Becton, Dickinson and Company/Sparks, MD21152 USA) plates and incubated at various temperatures $\left(30,35,37^{\circ} \mathrm{C}\right)$ in the dark for 3 weeks. The petri dishes were centrally inoculated with $10 \mu \mathrm{l}$ of the conidial suspension which was adjusted to 2.0-2.2 McFarland unit for each isolate, dried fully, and then placed upside down. The colony diameters (in $\mathrm{mm}$ ) were measured after 21 days of incubation. The microscopic features were determined primarily from slide cultures made on Corn Meal Agar (CMA-Oxoid Ltd, Basingstoke, Hampshire, England) after 10-12 days of incubation at $30{ }^{\circ} \mathrm{C}$. Coverslips were mounted in lactic acid and examined under a light microscope (Leica DM5000, German). The widths and lengths of the conidia were measured for each isolate. Dimorphism was demonstrated by conversion to the yeast-like form on Brain Heart Infusion (BHI-Bacto ${ }^{\mathrm{TM}}$ Becton, Dickinson and Company/Sparks, MD21152 USA) agar medium for 7 days at $37{ }^{\circ} \mathrm{C}$.

\section{Physiologic studies}

Carbohydrate assimilation tests were performed using freshly prepared yeast nitrogen base (YNB) medium (Difco $^{\mathrm{TM}}$ Becton, Dickinson and Company/Sparks, MD21152 USA) and tested for dextrose, sucrose, and raffinose according to methods described previously [1]. Cultures on YNB supplemented with dextrose were used as a positive control for growth, and YNB without carbohydrates was used as a negative control. Experiments were performed twice. The tests were performed in 96-well microplates. Microplates were read after 5 days of incubation at $30{ }^{\circ} \mathrm{C}$.

\section{Molecular identification}

Genomic DNA was extracted and purified from Sporothrix spp. mycelial phase by phenol/chloroform/isoamyl alcohol method with lysis step for 20 min with glass beads [11]. Amplification of the partial calmodulin-encoding (CAL) gene was performed [12] with degenerated primers CL1-GA(GA)T (AT)CAAGGAGGCCTTCTC and CL2A-TTTTTGC ATCATGAGTTGGAC as described by O'Donnell 
Table 1 Isolate, origin, clinical type, species, and GenBank accession numbers of Sporothrix isolates used in this study

\begin{tabular}{|c|c|c|c|c|}
\hline Isolates $^{\mathrm{a}}$ & Origin & Clinical type & Species $^{\mathrm{b}}$ & GenBank \\
\hline DMU1 & Clinical, Liaoning, China & Disseminated & S. globosa & $\mathrm{KC} 121564^{\mathrm{c}}$ \\
\hline DMU2 & Clinical, Liaoning, China & Lymphocutaneous & S. globosa & $\mathrm{KC} 190222^{\mathrm{c}}$ \\
\hline DMU3-DMU12 & Clinical, Liaoning, China & Lymphocutaneous & S. globosa & This study \\
\hline DMU13-DMU21 & Clinical, Liaoning, China & Fixed & S. globosa & This study \\
\hline DMU22 & Clinical, Liaoning, China & Fixed & S. globosa & $\mathrm{KC} 190217^{\mathrm{c}}$ \\
\hline HMU1 & Clinical, Heilongjiang, China & Lymphocutaneous & S. globosa & $\mathrm{KC} 121565^{\mathrm{c}}$ \\
\hline HMU2-HMU5 & Clinical, Heilongjiang, China & Lymphocutaneous & S. globosa & This study \\
\hline HMU6-HMU7 & Clinical, Heilongjiang, China & Fixed & S. globosa & This study \\
\hline HMU8 & Clinical, Heilongjiang, China & Fixed & S. globosa & $\mathrm{KC} 190221^{\mathrm{c}}$ \\
\hline HMU9-HMU10 & Clinical, Heilongjiang, China & Fixed & S. globosa & This study \\
\hline FHJU1 & Clinical, Jilin, China & Disseminated & S. globosa & $\mathrm{KC} 121566^{\mathrm{c}}$ \\
\hline FHJU2 & Clinical, Jilin, China & Disseminated & S. globosa & $\mathrm{KC} 190220^{\mathrm{c}}$ \\
\hline SHJU1 & Clinical, Jilin, China & Fixed & S. globosa & $\mathrm{KC} 121567^{\mathrm{c}}$ \\
\hline SHJU2 & Clinical, Jilin, China & Fixed & S. globosa & $\mathrm{KC} 190218^{\mathrm{c}}$ \\
\hline SHJU3-SHJU17 & Clinical, Jilin, China & Fixed & S. globosa & This study \\
\hline SHJU18-SHJU39 & Clinical, Jilin, China & Lymphocutaneous & S.globosa & This study \\
\hline SHJU40 & Clinical, Jilin, China & Lymphocutaneous & S. globosa & $\mathrm{KC} 190219^{\mathrm{c}}$ \\
\hline CBS120342 & Environmental, Mexico & & S. mexicana & AM398392 \\
\hline CBS120341 ${ }^{\mathrm{T}}$ & Environmental, Mexico & & S. mexicana & AM398393 \\
\hline $\mathrm{CBS} 302.73^{\mathrm{T}}$ & Environmental, UK & & S. pallida & AM398396 \\
\hline ATCC18616 & Clinical, South Africa & NK & S. luriei & AM747302 \\
\hline CBS120339 & Clinical, Brazil & NK & S. brasiliensis & AM116899 \\
\hline CBS359.36 & Clinical, USA & NK & S. schenckii & AM117437 \\
\hline CBS120340 ${ }^{\mathrm{T}}$ & Clinical, Spain & NK & S. globosa & AM116908 \\
\hline KMU4200 & Reed leaves, China & & S. globosa & AM399004 \\
\hline KMU4208 & Cornstalks, China & & S. globosa & AM399002 \\
\hline KMU4210 & Soil, China & & S. globosa & AM399005 \\
\hline CBS292.55 & Clinical, UK & NK & S. globosa & AM490354 \\
\hline IHEM4178 & Clinical, Italy & NK & S. globosa & AM399018 \\
\hline IPEC27135 & Clinical, Brazil & Lymphocutaneous & S. globosa & GU456632 \\
\hline FMR9020 & Clinical, Japan & NK & S. globosa & AM398994 \\
\hline MCCL220029 & Clinical, India & NK & S. globosa & AM490358 \\
\hline UTHSC04-1485 & Clinical, USA & NK & S. globosa & AM399015 \\
\hline
\end{tabular}

a Abbreviations: DMU The 1st Affiliated Hospital of Dalian Medical University; $H M U$ The Second Hospital of Harbin Medical University; FHJU The First Hospital of Jilin University; SHJU The Second Hospital of Jilin University; CBS Centraalbureau voor Schimmelcultures, Utrecht, the Netherlands; ATCC American Type Culture Collection; KMU Kanazawa Medical University, Ishikawa, Japan; IHEM, BCCM/IHEM Biomedical Fungi and Yeasts Collection, Belgium; IPEC Instituto de Pesquisa Clínica Evandro Chagas, Fiocruz, Brazil; FMR Facultat de Medicina i Ciències de la Salut, Reus, Spain; MCCL Mycology Culture Collection Laboratory, Postgraduate Institute of Medical Education and Research, Chandigarh, India; UTHSC Fungus Testing Laboratory, University of Texas Health Science Center; ${ }^{\mathrm{T}}$ type strain; $N K$ not known

b Identification based on calmodulin gene analysis

c The sequence was obtained in this study

et al. [13]. Automated sequencing was done at Sangon Biotech (Shanghai, China) with the same primers used for PCR.
The sequences of all our isolates were compared by BLAST (http://www.ncbi.nlm.nih.gov/blast) with several published Sporothrix-calmodulin-related sequences 
available from NCBI GenBank (AM398393/AM398392/ AM398396/AM747302/AM116899/AM117437/AM3 99018/AM490354/AM399004/AM399002/GU456632/ AM116908/AM398994/AM490358/AM399005/AM39 9015) (Table 1). Computer-assisted multiple sequence comparisons were made using ClustalW algorithm implemented in MEGA4 software [14]. The multiple nucleotide sequence alignment was inspected, visually adjusted, and subsequently was used for neighbor-joining analysis performed using MEGA4 software [14], and confidence was estimated using 1,000 rounds of bootstrapping.

Ten representative nucleotide sequences: $\mathrm{KC} 1215$ 64 (Isolate DMU1), KC121565 (Isolate HMU1), KC 121566 (Isolate FHJU1), KC121567 (Isolate SHJU1), KC190217 (Isolate DMU22), KC190218 (Isolate SHJU2), KC190219 (Isolate SHJU40), KC190220 (Isolate FHJU2), KC190221 (Isolate HMU8), and KC190222 (Isolate DMU2) have also been submitted to GenBank database.

\section{Results}

Morphological characterization

The macroscopic morphologies of all isolates were similar. After 21 days of incubation, colonies were pale orange to gray-orange on PDA with many wrinkles. All isolates showed temperature dimorphism with the typical cigar-shaped form of the yeastlike cells on brain heart infusion (BHI) agar medium for 7 days at $37{ }^{\circ} \mathrm{C}$. All isolates when cultured on corn meal agar for 12 days at $30{ }^{\circ} \mathrm{C}$ developed interim or terminal conidia in sympodial conidiophores along the hyphae. These conidia were hyaline to subhyaline, obovoidal or pear-shaped. In addition, sessile conidia were also observed, which were brown to dark brown, thick-walled, and connected individually throughout the hyphae, predominantly globose to subglobose, and 2.3-3.4 $\mu \mathrm{m}$ long by $1.9-2.8 \mu \mathrm{m}$ wide (Fig. 1). After incubation, respectively, at 30,35 , and $37{ }^{\circ} \mathrm{C}$ in the dark for 3 weeks, the best fungal growth was observed at $30{ }^{\circ} \mathrm{C}$, and the colonies attained a diameter of 25-45 mm with the mean colony diameter of $34 \mathrm{~mm}$. Colony growth at $35^{\circ} \mathrm{C}$ was lower than that observed at $30{ }^{\circ} \mathrm{C}$, with $12-15 \mathrm{~mm}$ diameter and mean $13 \mathrm{~mm}$ diameter. At $37^{\circ} \mathrm{C}$, most isolates showed restricted growth (up to mean $9 \mathrm{~mm}$ in diameter) (Fig. 2), with the exception of three isolates (SHJU28, DMU3, DMU20) which were unable to grow at $37{ }^{\circ} \mathrm{C}$.

Physiologic characterization

Carbohydrate assimilation tests were run in double and presented the following results: all isolates assimilated dextrose and sucrose, and were unable to assimilate raffinose, and none was able to grow on controls without carbohydrates.

\section{Molecular analysis}

The primers CL1 and CL2A were used to amplify a fragment of approximately 770 base pairs (bp) of the CAL gene (Fig. 3). The sequences blast revealed all isolates had a high level of sequence similarity with
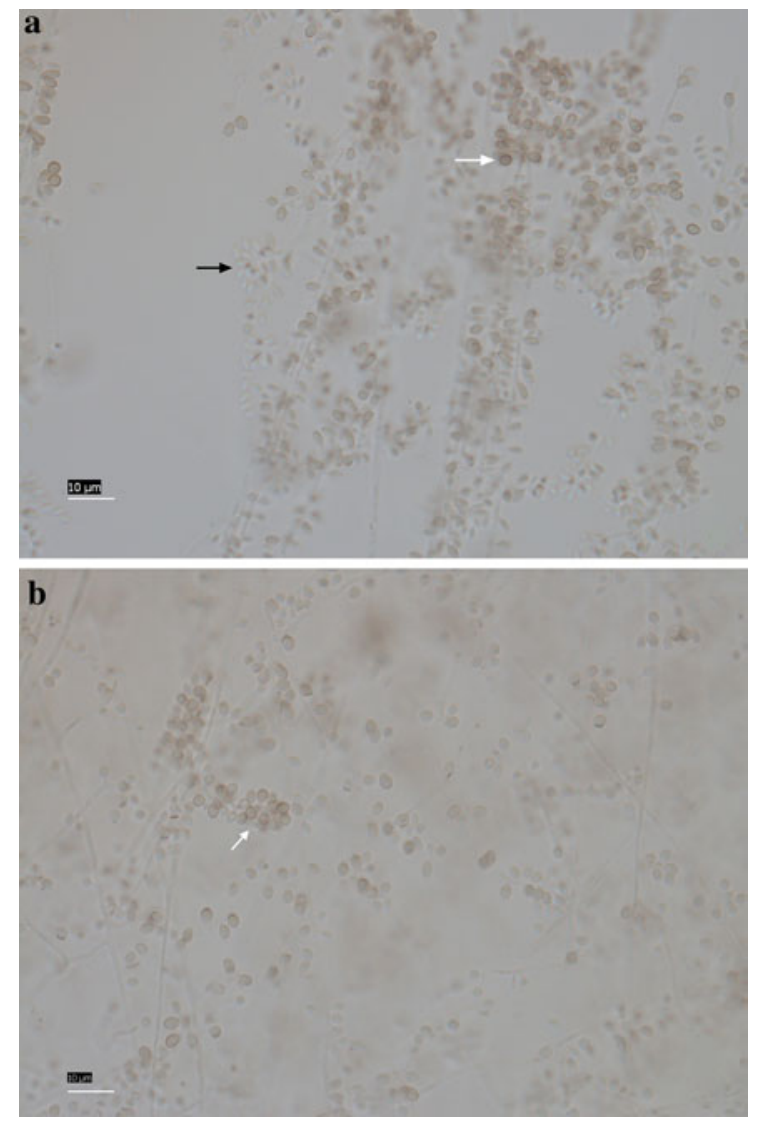

Fig. 1 Morphology of conidia of the S. globosa. a Black arrow subhyaline, obovoidal conidia in sympodial conidiophores, white arrow dark, globose, and sessile conidia connected individually. b White arrow dark, globose, and sessile conidia connected individually. Bars $10 \mu \mathrm{m}$ 


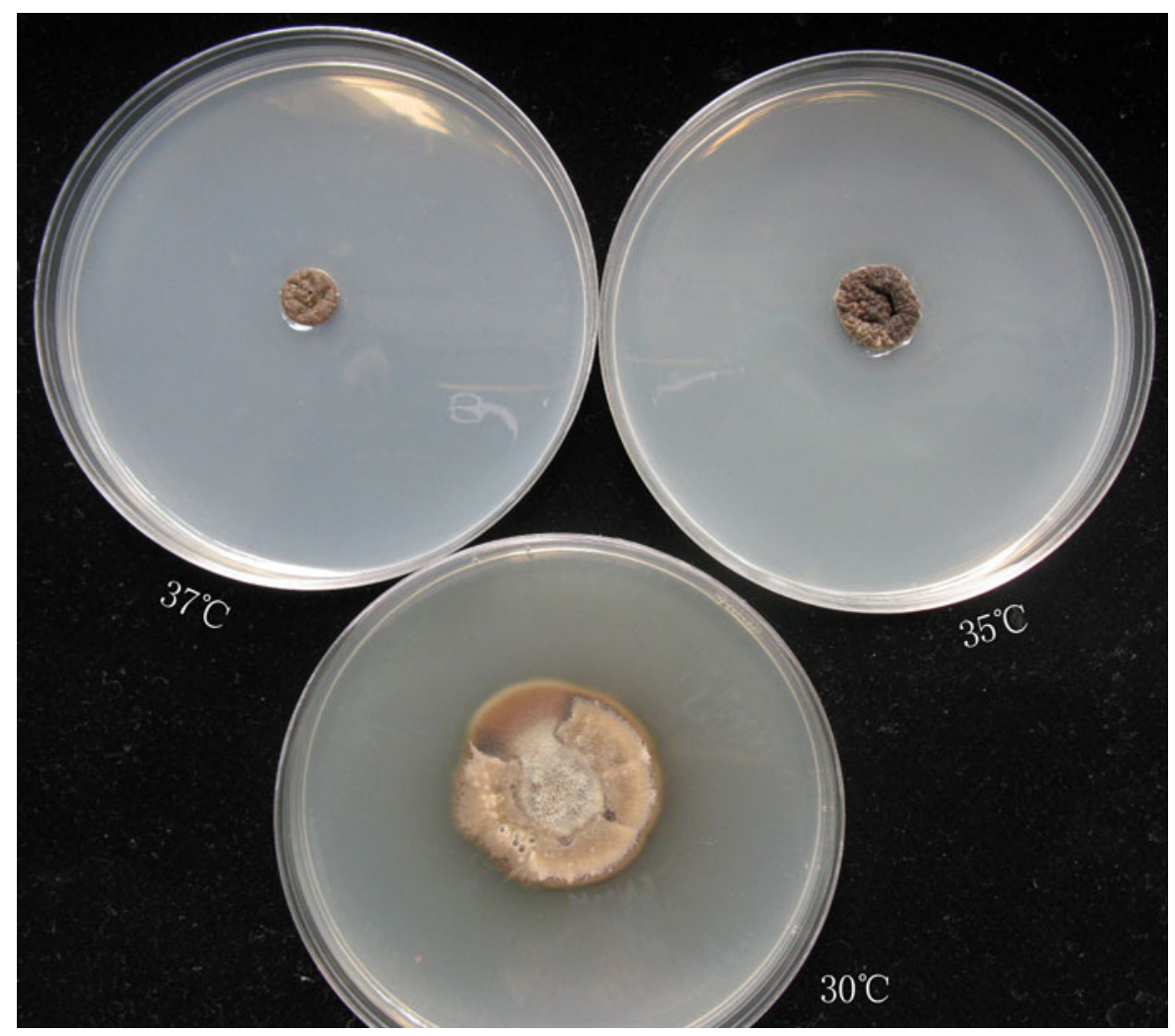

Fig. 2 Colony of $S$. globosa developed on PDA at 30,35 and $37^{\circ} \mathrm{C}$, respectively, in 21 days

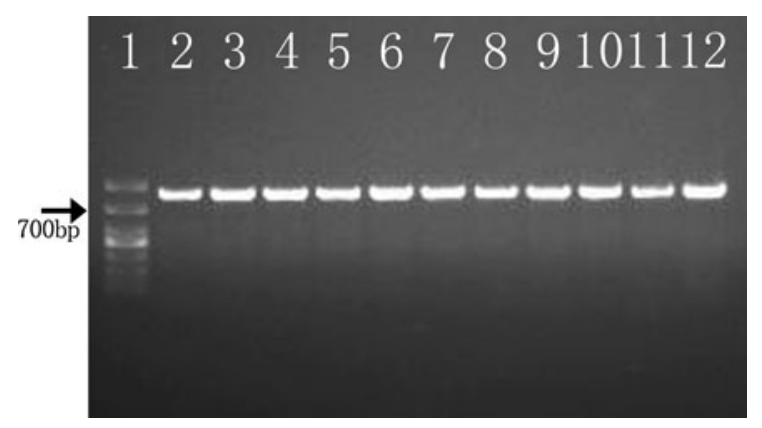

Fig. 3 PCR product based on the partial calmodulin gene sequence with CL1-CL2A primers pair. Lane 1 DL1000 markerTakara; Lanes 2-12 Sporothrix isolates: DMU1, DMU2, DMU22, HMU1, HMU2, HMU7, FHJU1, FHJU2, SHJU1, SHJU2, and SHJU40, respectively

previously published $S$. globosa strains listed in Table 1 (99-100 \%). The phylogenetic tree of the CAL locus analyzed by neighbor joining-NJ method-revealed six well-defined and supported groups (Fig. 4) as previously described [6]. All clinical isolates originated in Northeast China in this study were clustered with group S. globosa (AM116908 and AM399018).
Furthermore, our results showed that $S$. globosa could be further subdivided into two sub-clades: 68 of our isolates were clustered with AM116908, and the other six isolates were clustered with AM399018.

\section{Discussion}

Our phylogenetic analysis showed that seventy-four clinical isolates originated in Northeast China were entirely clustered with $S$. globosa. Moreover, these isolates were divided into two highly supported subclades (S. globosa I and S. globosa II). S. globosa I grouped sixty-eight Chinese clinical isolates, three previously published Chinese environmental isolates (AM399002, AM399005, and AM399004), the type strain of S. globosa (AM116908 from Spain), and some isolates from the USA (AM399015), India (AM490358), Japan (AM398994), Brazil (GU456632), UK (AM49 0354), while $S$. globosa II included six Chinese clinical isolates and the isolate from Italy (AM399018). S. globosa I was significantly more frequent than 
Fig. 4 Phylogenetic tree generated by neighborjoining analysis using partial nucleotide sequences of the calmodulin-encoding gene. Bootstrap support values above $85 \%$ are indicated at the nodes

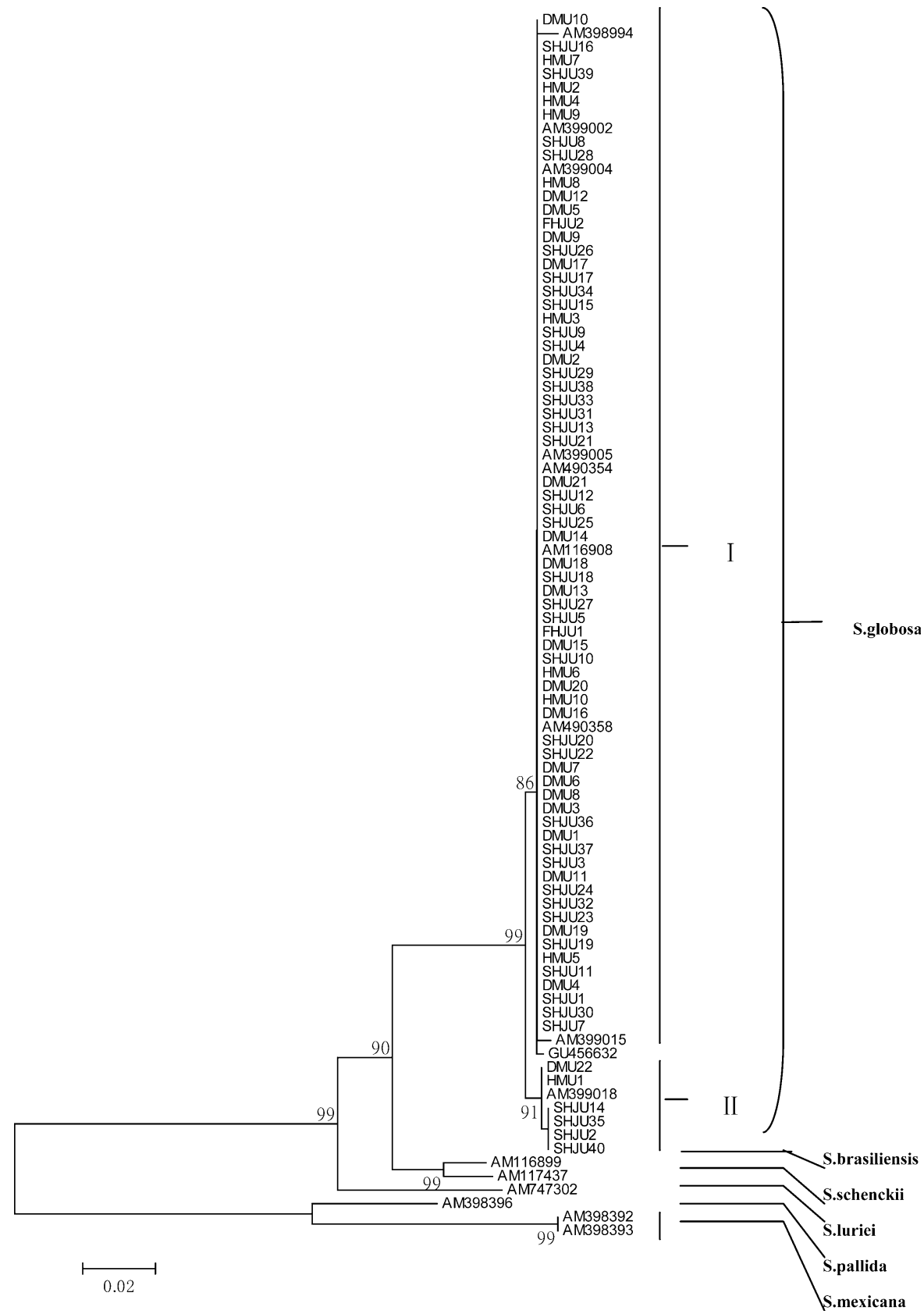

S. globosa II. The division of S. globosa was not related to geography, since the main clade of our clinical isolates, $S$. globosa I was more closely related to isolates from USA, Japan, and Brazil than to the other Chinese clinical isolates of $S$. globosa II. Sixty-eight Chinese clinical isolates were closely related to AM399002, AM399004, and AM399005, which were isolated from environment of Northeast China by Ishizaki and Xuezhu Jin [15]. Among them, AM399002 was from cornstalks, while AM399004 was from reed leaves and AM399005 was from soil [15], which indicated that most clinical isolates in Northeast China originated from autochthonous environment. Only six isolates were related to AM399018, which was isolated from Italy by Viviani in 1986 [16]. This suggested that a small amount of isolates in Northeast China were allochthonous. There was no association between the clinical form of sporotrichosis and the division of S. globosa. The origins of S. globosa I 
isolates in this study were fixed cutaneous $(n=29)$, lymphocutaneous $(n=36)$, and disseminated cutaneous $(n=3)$. S. globosa II isolates in this study were from fixed cutaneous $(n=3)$ and lymphocutaneous $(n=3)$ cases.

Although these clinical isolates were divided into two groups, they showed the same morphological and physiological features. They produced not only obovoidal, hyaline, sympodial conidia but also globose to subglobose, pigmented, sessile conidia. They were able to assimilate sucrose and unable to assimilate raffinose. The colonies of these isolates when grown on PDA attained a diameter of $25-45 \mathrm{~mm}$ at $30{ }^{\circ} \mathrm{C}$ in 21 days (not exceeding $50 \mathrm{~mm}$ ). According to the Marimon's key phenotypic features for species differentiation [1], the aforementioned phenotypic aspects are characteristic of S. globosa. However, we also noticed an important discrepancy with the results reported by Marimon et al. [1] with regard to the ability of $S$. globosa isolates to grow at $37{ }^{\circ} \mathrm{C}$. Marimon et al. proposed $S$. globosa did not present growth at $37{ }^{\circ} \mathrm{C}$. By contrast, most of our $S$. globosa isolates presented growth at $37{ }^{\circ} \mathrm{C}$, attaining $9 \mathrm{~mm}$ of colony diameter on PDA at $37^{\circ} \mathrm{C}$ in 21 days. Nevertheless, Marimon et al. related four exceptions, which exhibited very restricted growth (up to $2 \mathrm{~mm}$ in diameter in 21 days). Moreover, the first $S$. globosa isolate in Brazil identified by Oliveira et al. [5] also presented growth at $37^{\circ} \mathrm{C}$, attaining $7 \mathrm{~mm}$ of colony diameter. Consequently, disagreement in thermotolerance suggested that there may be some variation within this species. It seemed that the capacity for growth at $37{ }^{\circ} \mathrm{C}$ was not helpful for species differentiation. The reason for the difference in diameter at $37{ }^{\circ} \mathrm{C}$ may be that we inoculated $10 \mu \mathrm{l}$ of conidial suspension, while both Marimon and Oliveira inoculated pieces of the fungus that were approximately $1 \mathrm{~mm}$ in diameter. In addition, KwonChung [17] discovered that strains causing fixed cutaneous sporotrichosis grow best at $35^{\circ} \mathrm{C}$, while those causing lymphocutaneous form grow at both 37 and $35{ }^{\circ} \mathrm{C}$. However, in our study, the isolates which were thermotolerant at $37{ }^{\circ} \mathrm{C}$ could be obtained from fixed cutaneous, lymphocutaneous, or disseminated sporotrichosis. Our observations were similar to those of Mehta et al. [18] who observed that isolates from both fixed and lymphocutaneous types grew well at $37^{\circ} \mathrm{C}$. These phenomena further hinted that difference in thermotolerance may be related to individual variation.

Based on the phenotypic and genetic analysis, we found that all clinical isolates studied belonged to the same species, i.e., S. globosa, independent of geographical regions and clinical forms of sporotrichosis, which suggested it was not Sporothrix species that determined types of clinical presentation. Our previous studies [19] showed that the isolate (Sp98-12-1, i.e. DMU1) from disseminated sporotrichosis presented 10-bp deletion in the ribosomal nontranscribed spacer (NTS) region and higher virulence compared to the isolate (D1, i.e. DMU15) from fixed sporotrichosis, which further suggested from the gene level that there may be some variation within this species. It appears that the strain variation in genotypes and virulence as well as immune status of the host may contribute to disseminated type of sporotrichosis. We also drew a conclusion that outbreaks of sporotrichosis in Jilin province over the past 2 years were caused by the same species as the sporadic cases in Heilongjiang and Liaoning provinces, which could be explained by the fact that in Northeast China, wide contact with contaminated cornstalks led to high incidence. The people living in rural areas accounted for a considerable proportion of sporotrichosis patients, who were used to stacking cornstalks for use in cooking or heating. Isolation of $S$. schenckii from cornstalks of Northeast China has been reported [15], which proves to be $S$. globosa now [1]. Over time, fungal growth on this material increases as the cornstalks decay and become the source of contamination. Outbreaks might be associated with increased chances of contact in autumn and winter or increased Sporothrix quantity in the environment, which was also confirmed by our results that most clinical isolates were clustered with Chinese environmental isolates.

Acknowledgments This work was completed in the Centre for Medical Mycology and Mycoses, First Hospital, Peking University. I am indebted to all the staff for help in this study. I am also indebted to Yuzhen Li (The Second Hospital of Harbin Medical University), Shanshan Li (The First Hospital of Jilin University), and Fuqiu Li (The Second Hospital of Jilin University) for supplying some isolates used in this study.

\section{Conflict of interest None.}

Open Access This article is distributed under the terms of the Creative Commons Attribution License which permits any use, distribution, and reproduction in any medium, provided the original author(s) and the source are credited. 


\section{References}

1. Marimon R, Cano J, Gené J, Sutton DA, Kawasaki M, Guarro J. Sporothrix brasiliensis, S. globosa, and S. mexicana, three new Sporothrix species of clinical interest. J Clin Microbiol. 2007;45:3198-206.

2. Marimon R, Gené J, Cano J, Guarro J. Sporothrix luriei: a rare fungus from clinical origin. Med Mycol. 2008;46: 621-5.

3. de Meyer EM, de Beer ZW, Summerbell RC, Moharram AM, de Hoog GS, Vismer HF, Wingfield MJ. Taxonomy and phylogeny of new wood- and soil-inhabiting Sporothrix species in the Ophiostoma stenoceras-Sporothrix schenckii complex. Mycologia. 2008;100:647-61.

4. Madrid H, Cano J, Gené J, Bonifaz A, Toriello C, Guarro J. Sporothrix globosa, a pathogenic fungus with widespread geographical distribution. Rev Iberoam Micol. 2009;26: 218-22.

5. Oliveira MME, Almeida-Paes R, Muniz MM, Barros MBL, Gutierrez-Galhardo MC, Zancopé-Oliveira RM. Sporotrichosis caused by Sporothrix globosa in Rio de Janeiro, Brazil: case report. Mycopathologia. 2010;169:359-63.

6. Romeo O, Scordino F, Criseo G. New insight into molecular phylogeny and epidemiology of Sporothrix schenckii species complex based on calmodulin-encoding gene analysis of Italian isolates. Mycopathologia. 2011;172:179-86.

7. Zhang J, Lin J. Clinical analysis of 316 cases of cutaneous sporotrichosis [in Chinese]. Chin J Mycol. 2008;3:207-10.

8. Song Y, Yao L, Zhong S, Tian Y, Liu Y, Li S. Infant sporotrichosis in northeast China: a report of 15 cases. Int $\mathbf{J}$ Dermatol. 2011;50:522-9.

9. Li S, Liu H, Zheng H, Chen S, Fu A, Wang H, Song Y, Yao L, Liu Y. Clinical analysis of 585 cases of cutaneous sporotrichosis [in Chinese]. Chin J Dermatol. 2011;44:161-4.

10. Mesa-Arango AC, Del Rocío Reyes-Montes M, Pérez-Mejía A, Navarro-Barranco H, Souza V, Zúniga G, Toriello C.
Phenotyping and genotyping of Sporothrix schenckii isolates according to geographic origin and clinical form of sporotrichosis. J Clin Microbiol. 2002;40:3004-11.

11. Criseo G, Romeo O. Ribosomal DNA sequencing and phylogenetic analysis of environmental Sporothrix schenckii strains: comparison with clinical isolates. Mycopathologia. 2010;169:351-8.

12. Marimon R, Gené J, Cano J, Trilles L, Dos Santos Lazéra M, Guarro J. Molecular phylogeny of Sporothrix schenckii. J Clin Microbiol. 2006;44:3251-6.

13. O’Donnell K, Nirenberg HI, Aoki T, Cigelnik E. A multigene phylogeny of the Gibberella fujikuroi species complex: detection of additional phylogenetically distinct species. Mycoscience. 2000;41:61-78.

14. Tamura K, Dudley J, Nei M, Kumar S. MEGA4: molecular evolutionary genetics analysis (MEGA) software version 4.0. Mol Biol Evol. 2007;24:1596-9.

15. Ishizaki H, Kawasaki M, Mochizuki T, Jin X, Kagawa S. Environmental isolates of Sporothrix schenckii in China. Jpn J Med Mycol. 2002;43:257-60.

16. Neyra E, Fonteyne PA, Swinne D, Fauche F, Bustamante B, Nolard N. Epidemiology of human sporotrichosis investigated by amplified fragment length polymorphism. J Clin Microbiol. 2005;43:1348-52.

17. Kwon-Chung KJ. Comparison of isolates of Sporothrix schenckii obtained from fixed cutaneous lesions with isolates from other types of lesions. J Infect Dis. 1979;139:424-31.

18. Mehta KIS, Sharma NL, Kanga AK, Mahajan VK, Ranjan N. Isolation of Sporothrix schenckii from the environmental sources of cutaneous sporotrichosis patients in Himachal Pradesh, India: results of a pilot study. Mycoses. 2007;50: 496-501.

19. Zhang Z, Liu X, Lv X, Lin J. Variation in genotype and higher virulence of a strain of Sporothrix schenckii causing disseminated cutaneous sporotrichosis. Mycopathologia. 2011;172:439-46. 